\title{
Récents progrès expérimentaux et théoriques en micro-perçage des polymères par faisceaux laser ultraviolets
}

\author{
Sylvain LAZARE ${ }^{a}$ ET Vladimir TOKAREV \\ Laboratoire de Physico-Chimie Moléculaire, LPCM UMR 5803, Université de Bordeaux 1, 351 cours de la Libération, \\ 33405 Talence, France
}

Reçu le 21 octobre 2004, accepté le 25 janvier 2005

\begin{abstract}
Résumé - L'utilisation du perçage laser s'avère une alternative incontournable lorsque le diamètre de perçage est aux environs de 10 à $50 \mu \mathrm{m}$, domaine où les moyens conventionnels deviennent difficiles à mettre en œuvre et coûteux. De plus il se présente comme une technique de choix pour un certain nombre de matériaux : les composites, les mous et les ultradurs, qui ne sont pas travaillés facilement au niveau microscopique avec les outils de coupe mécaniques. Nous avons apporté la preuve que des conditions d'expérience bien choisies sont capables de produire des micro-trous avec des rapports de forme records (jusqu'à 600) dans des polymères purs comme les PET, PI, PC, PS, PMMA, PEEK,... Par exemple des trous de diamètre typiquement dans les $30 \mu \mathrm{m}$ peuvent être aussi longs que $18 \mathrm{~mm}$, profondeur à laquelle la vitesse tombe presque à zéro, et le profil devient stationnaire. D'autres matériaux (métaux, céramiques), qui peuvent être de la même façon micropercés par laser, n'offrent pas les mêmes rapports de forme aussi élevés. Les mécanismes du processus de perçage ont été étudiés en détail et un modèle analytique original a été construit récemment. Les divers résultats expérimentaux, obtenus avec le laser $\mathrm{KrF}$, sont revus en insistant sur les paramètres conduisant à la formation de «bons » trous avec les rapports de forme élevés. Pour les applications il est aussi important de noter que de tels rapports de forme élevés sont obtenus avec la configuration ordinaire du laser $\mathrm{KrF}$ ayant une divergence de $3 \mathrm{mrad}$ en standard. Cependant, comme le modèle le montre, il y a encore de l'espace pour une amélioration du rapport de forme en utilisant un faisceau avec une divergence plus faible (la limite théorique est 0,2 mrad). De nouveaux travaux de recherche se poursuivent actuellement.
\end{abstract}

Mots clés : Matériaux / laser / usinage / perçage / modèle / profil / mécanismes / polymère / divergence / découpe

\begin{abstract}
Recent experimental and theoretical advances in microdrilling of polymers with ultraviolet laser beams. Laser drilling becomes of increasing importance when hole diameter is in the range of 10 to $50 \mu \mathrm{m}$, for which conventional alternative approaches are becoming difficult and cost inefficient. Furthermore, it is viewed as the technique of choice for a number of composite materials and hard materials which are not readily processed at the microscopic level by contact mechanical tools. We have demonstrated that suitable experimental conditions are capable of producing microholes with record aspect-ratio (up to 600) in pure polymers like PET, PI, PC, PS, PMMA, PEEK,... For example holes of diameter typically $30 \mu \mathrm{m}$ can be as long as $18 \mathrm{~mm}$, depth at which the drilling rate is getting nearly zero and the profile stationary. Other materials (metals, ceramics) which can be similarly laser microdrilled do not exhibit such very high aspect-ratio. The mechanisms of the drilling process have been studied in details and an original analytical model has been constructed recently. The various experimental results, obtained with the $\mathrm{KrF}$ laser, will be reviewed with emphasis on the parameters leading to formation of good holes with high aspect-ratio. For the application it is also important to note that such high values of aspect-ratio are obtained with regular configuration of the $\mathrm{KrF}$ laser giving a standard divergence of $3 \mathrm{mrad}$. However as shown by the model there is still room for improvement by using a beam with a lower divergence (theoretical limit is $0.2 \mathrm{mrad}$ ). Further experimental work is now in progress.
\end{abstract}

Key words: Materials / laser / processing / drilling / model / profile / mechanisms / polymer / divergence / cutting

\footnotetext{
${ }^{a}$ Auteur correspondant : s.lazare@lpcm.u-bordeaux1.fr
} 


\section{Nomenclature}

\begin{tabular}{|c|c|c|}
\hline$A_{\text {eff }}, A(i)$ & absorptivité à la surface à l'incidence $i$ & \\
\hline$D_{r}$ & vitesse d'ablation (profondeur par impulsion) & $\mu \mathrm{m} \cdot \mathrm{pls}^{-1}$ \\
\hline$d$ & diamètre du trou & $\mu \mathrm{m}$ \\
\hline$d_{\mathrm{w}}$ & diamètre du waist & $\mu \mathrm{m}$ \\
\hline E & énergie d'une impulsion du laser & $\mathrm{J}$ \\
\hline$f$ & distance focale de la lentille de projection & $\mathrm{mm}$ \\
\hline$f_{\text {cond }}$ & distance focale de la lentille de condensation & $\mathrm{mm}$ \\
\hline$F, F_{0}$ & fluence du laser (énergie par $\mathrm{cm}^{2}$ et par impulsion) & J.cm ${ }^{-2}$ \\
\hline$F_{\mathrm{a}}$ & fluence absorbée par le matériau & $\mathrm{J} . \mathrm{cm}^{-2}$ \\
\hline$F_{\mathrm{c}}$ & fluence caractéristique du polymère & $\mathrm{mJ} . \mathrm{cm}^{-2}$ \\
\hline$F_{\text {par }}$ & fluence donnant un trou avec des parois parallèles à l'entrée & \\
\hline$F_{\mathrm{t}}$ & fluence seuil d'ablation & $\mathrm{mJ} . \mathrm{cm}^{-2}$ \\
\hline$F_{\infty}$ & $\begin{array}{l}\text { fluence seuil d'arrachement de matière } \\
\text { après un grand nombre d'impulsions }\end{array}$ & \\
\hline$I$ & intensité instantanée du laser & $\mathrm{W}$ \\
\hline$i$ & incidence de la radiation & $\circ$ \\
\hline$l$ & longueur du trou & $\mathrm{mm}$ \\
\hline$r, r(z)$ & rayon du trou à la profondeur $z$ & $\mu \mathrm{m}$ \\
\hline$r_{0}$ & rayon d'entrée du trou & $\mu \mathrm{m}$ \\
\hline$R, R_{m}=l / d$ & rapport de forme & \\
\hline$s_{0}$ & surface d'entrée du trou conique & $\mu \mathrm{m}^{2}$ \\
\hline$s$ & surface latérale du trou conique & $\mu \mathrm{m}^{2}$ \\
\hline$T(z)$ & transmission du laser à la profondeur $z$ & \\
\hline$w_{\alpha}$ & paramètre de la fonction gaussienne & \\
\hline$z$ & $\begin{array}{l}\text { coordonnée axe du trou, origine en } \mathrm{O} \text { et positive } \\
\text { vers l'intérieur du trou }\end{array}$ & \\
\hline$z_{0}$ & distance de la source ponctuelle à la surface de l'échantillon & \\
\hline \multicolumn{3}{|c|}{ Symboles grecs } \\
\hline$\alpha$ & inclinaison des rayons par rapport à l'axe $z$ du trou & \\
\hline$\alpha^{\prime}$ & conicité du trou & \\
\hline$\alpha_{\mathrm{c}}$ & demi-angle du trou conique (Fig. 5) & \\
\hline$\alpha_{\infty}$ & angle limite d'intégration & \\
\hline$\beta$ & angle inclinaison de la surface du trou par rapport à son axe & \\
\hline$\gamma$ & coefficient d'absorption & $\mu \mathrm{m}^{-1}$ \\
\hline$\delta$ & défocus, distance image surface & $\mu \mathrm{m}$ \\
\hline$\phi(r, z)$ & $\begin{array}{l}\text { inclinaison d'un rayon par rapport à la tangente au point } \mathrm{P}(r, z) \\
\text { à la surface du trou }\end{array}$ & \\
\hline$\lambda$ & longueur d'onde du laser & $\mathrm{nm}$ \\
\hline$\nu$ & fréquence de la lumière laser & $\mathrm{s}^{-1}$ \\
\hline$\tau$ & durée d'impulsion (25 ns) & ns \\
\hline \multicolumn{3}{|l|}{ Indices } \\
\hline eff & effectif, pour le coefficient d'absorption & \\
\hline $\mathrm{a}$ & absorbée, pour la fluence du laser & \\
\hline $\mathrm{t}$ & seuil d'ablation (threshold) généralement pour une impulsion & \\
\hline$\infty$ & seuil d'extinction de l'ablation, après un grand nombre d'impulsions & \\
\hline
\end{tabular}




\section{Introduction}

Le microperçage est l'une des étapes clés pour les futures technologies dans l'industrie. Le perçage mécanique conventionnel doit faire face à des limites physiques grandissantes lorsque le diamètre du trou diminue. En dessous d'une certaine dimension critique, la friction dépasse la résistance mécanique de l'outil et l'usinage ne devient possible que grâce à l'emploi d'outils spéciaux faits de matériaux à haut module (par exemple diamant, cBN, etc.). De plus, le temps d'usinage peut être rallongé et la durée de vie de l'outil peut être sacrifiée. Dans la plupart des cas, cela veut dire que le coût du traitement augmente de façon prohibitive. Dans ce contexte le microperçage laser [1-12] apparaît comme une bonne alternative à l'usinage mécanique. Il est d'ailleurs très utilisé dans les applications : par exemple pour des conduits microscopiques de fluides gazeux, liquides ou solides (microfluidique). Respectivement on peut citer les capteurs de gaz, les éléments de raréfaction de la couche limite en aéronautique, les implants oculaires en ophtalmologie, les injecteurs de carburant en automobile, les filières de diamant, etc. De plus, le même outil laser peut usiner à l'identique n'importe quel matériau, indépendamment de ses propriétés physiques (dureté, mollesse, composite, absorptivité...), bien que les mécanismes d'arrachement de matière puissent être très divers. Dans ce cadre nous avons récemment montré les performances ultimes du microperçage par laser [13-17] principalement dans des matériaux polymères. En particulier l'attention a été portée sur les deux dimensions d'intérêt pour les applications de microperçage, le diamètre du trou $(d)$ et sa longueur $(l)$ obtenus pour un ensemble donné de paramètres laser (longueur d'onde, taille de faisceau, densité d'énergie). Dans ce travail, nous montrons que des rapports de forme jamais réalisés ( $R=l / d$ jusqu'à 600$)$ peuvent être obtenus lorsque les conditions expérimentales sont bien choisies. Nous avons expérimenté avec des polymères, mais les résultats peuvent être transposés aux cas d'autres matériaux (céramiques, métaux,...) avec plus ou moins de complications. La première considération est donnée par l'équation (1) qui exprime le diamètre du faisceau $d_{\mathrm{w}}$ après la transformation d'un faisceau laser gaussien de diamètre initial $D$ par une lentille simple (Fig. 1) de focale $f[18]$ :

$$
d_{\mathrm{w}}=4 \lambda f / \pi D
$$

où $\lambda$ est la longueur d'onde du laser.

Un faisceau de petit diamètre est favorisé en choisissant un laser de longueur d'onde courte et une petite focale. Pour illustrer ceci : un faisceau de $3 \mu \mathrm{m}$ est obtenu avec un laser $\operatorname{KrF}(\lambda=248 \mathrm{~nm})$ avec une lentille de distance focale $20 \mathrm{~mm}$ pour une taille de faisceau incident de $1 \mathrm{~mm}$. Pour comparaison le faisceau d'un laser infrarouge à $\mathrm{CO}_{2}(\lambda=10,6 \mu \mathrm{m} \approx 43 \times 0,248 \mu \mathrm{m})$ donnerait un spot 43 fois plus large $(d=120 \mu \mathrm{m})$. Donc pour la même énergie laser initiale la fluence au point focal serait en conséquence beaucoup plus faible $\left(\sim\left(\lambda_{\mathrm{KrF}} / \lambda_{\mathrm{CO} 2}\right)^{2} \approx 1 / 1850\right)$ parce qu'étalée sur une surface plus grande. En conséquence les lasers ultraviolets

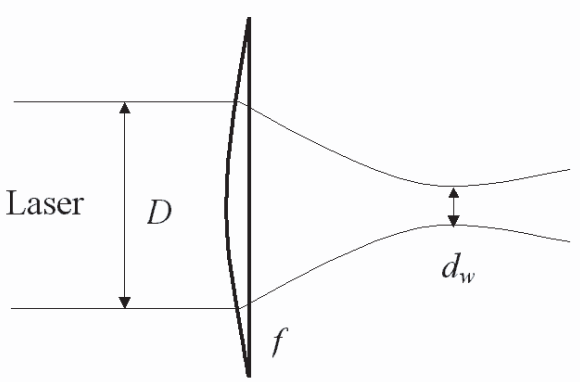

Fig. 1. Transformation du faisceau par une lentille simple.

sont de meilleurs candidats afin de réaliser des spots plus petits avec une densité d'énergie plus grande pour obtenir des microperçages à haut rapport de forme par ablation profonde. De plus en UV les seuils d'ablation sont faibles parce que les coefficients d'absorption $\gamma$ sont élevés $\left(10^{5} \mathrm{~cm}^{-1}\right)$. Donc les densités d'énergie seuil d'ablation sont facilement dépassées et le grand excès, par rapport aux valeurs seuil, fournit l'énergie cinétique nécessaire pour l'expulsion du matériau ablaté vers l'extérieur du microperçage profond. Dans le présent état de l'art, les lasers $\mathrm{KrF}$ ayant une puissance convenable $\left(0,4 \mathrm{~J}_{\text {.pulse }}{ }^{-1}\right.$, $200 \mathrm{~Hz}$ ) sont utilisés parce que le microperçage est exigeant en nombre d'impulsions et d'autres lasers à longueur d'onde plus courte (par ex. ArF ou VUV) sont aussi envisageables bien qu'ayant moins d'énergie par impulsion et une fréquence de tir plus faible. Il est aussi envisageable que dans le futur, des lasers de longueur d'onde encore plus courte (UV lointain, rayon X) pourront être utilisés en mettant à profit leur grand potentiel de focalisabilité. Un autre avantage de la radiation ultraviolette est le grand contenu énergétique de chaque photon (e.g. $h \nu=5 \mathrm{eV}$ pour $248 \mathrm{~nm}$ ) qui est assez grand pour engendrer la cassure directe des liaisons covalentes par absorption monophotonique. Les polymères sont des molécules à longues chaînes. Une partie de l'énergie incidente est convertie en chaleur pouvant donner lieu à des transitions de phase comme solide-versliquide et des mécanismes complexes d'expulsion de liquide peuvent aussi avoir lieu. Les lasers de longueur d'onde plus grande, ayant une énergie de photon plus petite que la moyenne des énergies de liaison, montrent une plus grande tendance à des mécanismes thermiques, avec une décomposition en molécules gazeuses moins directe, et un volume affecté plus grand autour du microperçage, comme expliqué plus bas. En conséquence bien qu'un microperçage efficace soit obtenu avec de tels lasers dans un vaste domaine d'applications, les meilleures performances en termes de précision microscopique (diamètre latéral) sont obtenues actuellement avec des lasers de courte longueur d'onde. L'efficacité pour le microperçage du laser ultraviolet d'énergie donnée, avec des impulsions nanoseconde, est alors due à la synergie entre la grande focalisabilité et la forte absorptivité en UV des matériaux solides donnant des seuils d'ablation faibles, qui rend possible l'éjection de la matière ablatée avec l'énergie 
cinétique importante nécessaire pour le perçage profond. Cet article fait une synthèse des travaux de ces dernières années [19] et il commence avec une description du dispositif expérimental en insistant sur les points stratégiques. À partir des résultats expérimentaux présentés à la section 2 ci-dessous, on verra que le rapport de forme maximal augmente avec la fluence comme prédit par le modèle présenté à la section 3. Le modèle prédit aussi le profil du trou, la transmission du faisceau dans le trou et la fluence seuil de perçage, qui pourrait bien être l'énergie minimale qui permet d'arracher de la matière à la surface après incubation et ablation avec un grand nombre d'impulsions. Dans la section 4 suivante, les mécanismes et les perspectives de cette étude seront discutés.

\section{L'expérience de microperçage}

Dans ces expériences, un bon microperçage est obtenu seulement lorsque le microfaisceau laser est de grande qualité sur la grande profondeur du trou recherché, c'està-dire : une grande densité d'énergie, un bon profil et de petites dimensions latérales. Le microperçage s'avère être lui-même un bon test pour la qualité de faisceau, utilisable pour l'expérimentateur pour ajuster le banc optique. Tout autre détecteur de photon (comme une caméra) est dans le cas présent inutile ou inefficace car la densité d'énergie utilisée est trop élevée et la taille du faisceau est trop petite.

\subsection{Banc optique du faisceau laser}

Le banc optique du faisceau laser est montré à la figure 2. Il est composé de deux lentilles plan-convexe en silice (Suprasil). L'une est le condenseur $\left(f_{\text {cond }}=250 \mathrm{~mm}\right)$ et l'autre est le projecteur $(f=25 \mathrm{~mm})$. Elles sont choisies de faible diamètre, pour garder l'épaisseur traversée par le faisceau au minimum, afin d'éliminer le plus possible d'éventuelles atténuations non désirées de la radiation (elles doivent supporter 200 tir.s ${ }^{-1}$ pendant des minutes avec une bonne stabilité). L'utilisation d'une lentille de précision comme optique de projection [20] n'est pas complètement exclue mais ne fut pas préférée pour l'étude présente. Le laser à excimère est un modèle Lambda Physik 220i (énergie par impulsion $E=0,4 \mathrm{~J}$, durée d'impulsion $\tau=25$ ns, intensité pic de l'impulsion $I=E / \tau=1,6 \times 10^{7} \mathrm{~W}$ et fréquence de tirs maximale $200 \mathrm{~Hz}$ ) dont la cavité optique est équipée de miroirs plans et de longueur 1,50 m, offrant ainsi un maximum d'énergie dans les modes les moins divergents du résonateur. Un masque circulaire en molybdène (e.g. $\Phi=250 \mu \mathrm{m})$ est utilisé pour la mise en forme du faisceau et est placé à une distance du condenseur égale approximativement à $f_{\text {cond }}$. Ce masque est imagé précisément sur la surface du polymère, comme illustré sur la figure 3 , à l'aide de la lentille de projection, de telle manière que le rapport de démagnification soit de l'ordre de $1 / 5$. Une grille fine de nickel (Goodfellow, période $340 \mu \mathrm{m}$ ) est placée sur le masque et la cible de polymère est déplacée au voisinage

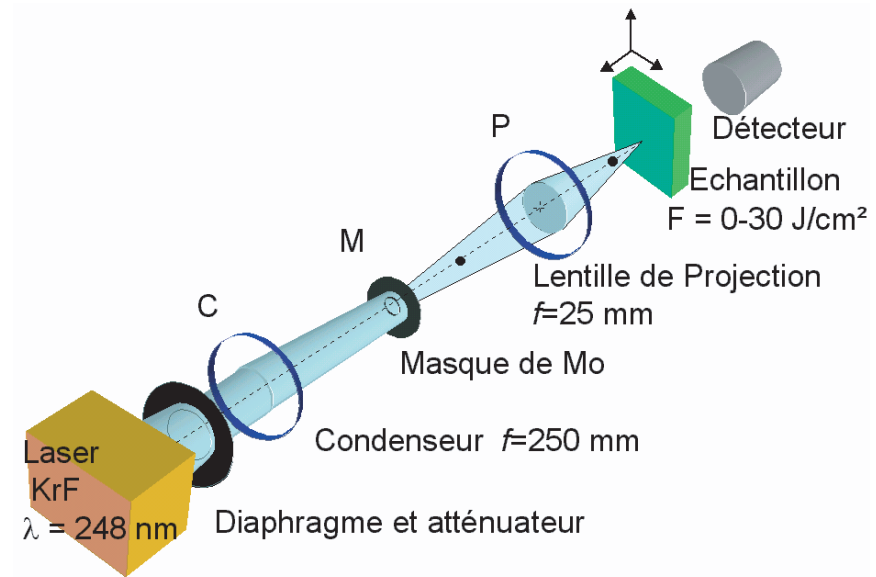

Fig. 2. Banc optique de mise en forme du laser $\mathrm{KrF}$.

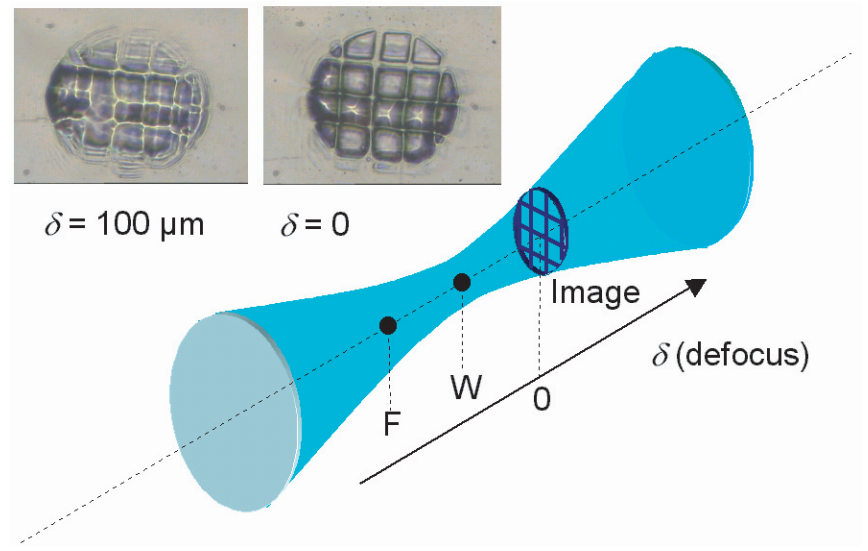

Fig. 3. Positionnement de l'échantillon dans le faisceau.

de la position du point le plus étroit du faisceau (waist) jusqu'à obtenir la bonne position donnant la meilleure image d'ablation (qui est appelée alors « defocus » zéro $\delta=0$ ) comme dans l'image de microscopie en insert en haut à gauche de la figure 3. Le polycarbonate est utilisé pour cet ajustement fin, réalisé avec une seule impulsion, car son ablation n'est pas perturbée par la formation d'un film liquide (comme pour le PET) et son écoulement latéral. Cette approche s'est révélée une technique de réglage très sensible. Deux diaphragmes sont aussi utilisés pour limiter l'influence de la radiation diffusée et sont positionnés avant chaque lentille. Leurs réglages s'effectuent sur la base d'une démarche empirique.

\subsection{Les échantillons de polymères}

Les échantillons de polymères sélectionnés furent choisis, dans une première série, à cause de leurs bonnes propriétés optiques et de surface (PC, PS, PMMA, PET) permettant une observation facile au microscope optique et dans une seconde série pour leur importance technologique (PI et PEEK). Les polymères étudiés sont des polyimides (PI), poly(éthylène téréphthalate) (PET), polystyrène (PS), poly(éther éther kétone) (PEEK), 
Tableau 1. Polymères : coefficients d'absorption $\gamma$, pour le laser KrF à $248 \mathrm{~nm}$, profondeur d'absorption $1 / \gamma$, seuil d'ablation $F_{\mathrm{t}}$ à $248 \mathrm{~nm}$ et paramètres de perçage : vitesse $D_{\mathrm{r}}$, rapport de forme maximal $R_{\mathrm{m}}$ et conicité du trou $\alpha^{\prime}$.

\begin{tabular}{ccccccc}
\hline Matériau & $\gamma\left(\mu \mathrm{m}^{-1}\right)$ & $1 / \gamma(\mu \mathrm{m})$ & $F_{\mathrm{t}}\left(\mathrm{mJ.cm}^{-2}\right)$ & $D_{\mathrm{r}}\left(\mu \mathrm{m} . \mathrm{impl} .^{-1}\right)$ & $R_{\mathrm{m}}=l / d$ & $\alpha^{\prime}=1 / R_{\mathrm{m}}\left(10^{-3}\right)$ \\
\hline PET & 16 & 0,065 & 30 & 0,7 & 565 & 1,77 \\
PC & 1 & 1,0 & 40 & 0,8 & 390 & 2,56 \\
PEEK & $\sim 10$ & $\sim 0,1$ & 50 & 0,6 & 385 & 2,60 \\
PI & 22 & 0,045 & 54 & 0,4 & 360 & 2,78 \\
PS & 0,61 & 1,6 & 40 & - & 315 & 3,17 \\
PMMA & 0,0063 & 150 & 250 & 2,5 & 255 & 3,92 \\
\hline
\end{tabular}

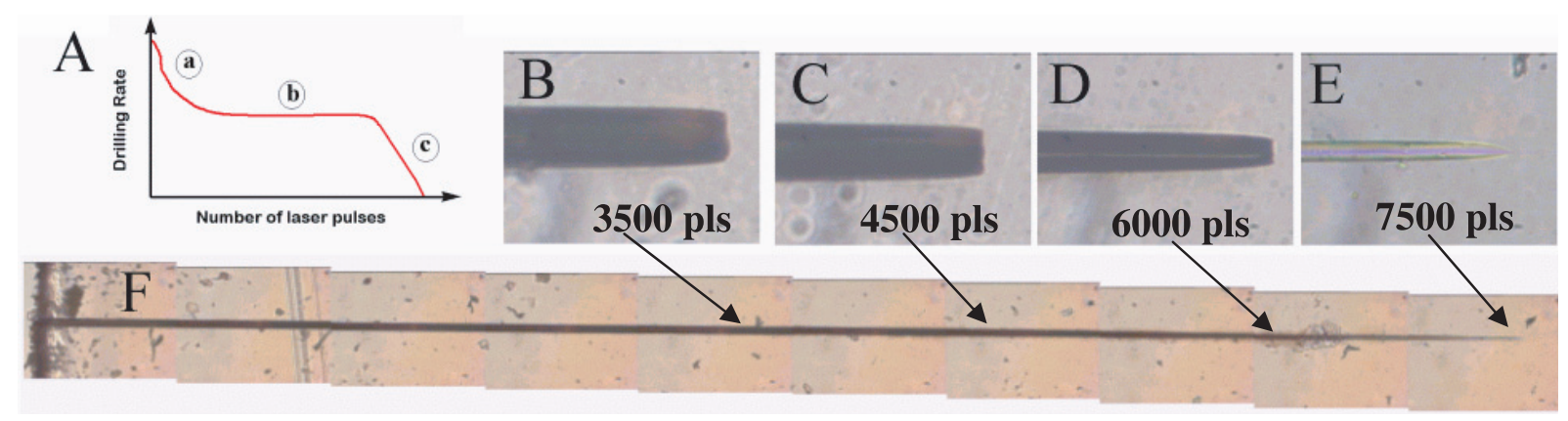

Fig. 4. Expérience typique de microperçage dans le PET avec le laser KrF. Diamètre $25 \mu \mathrm{m}$ et longueur 5 mm. A vitesse de perçage en fonction de l'avancement du processus. B à $\mathbf{E}$ profil du front principal d'ablation à différents temps de l'expérience. $\mathbf{E}$ et $\mathbf{F}$ trou après le profil stationnaire (fin du microperçage), $\mathbf{E}$ pointe finale. L'échelle de l'image est donnée par le diamètre du trou et la vue générale est le résultat de l'assemblage de dix vues partielles au microscope optique.

poly(méthyle métacrylate) (PMMA) et bis-phénol A polycarbonate (PC). Les noms commerciaux sont Kapton pour PI, Mylar pour PET, Stabar pour PEEK. Les échantillons de PS ont été pris à partir de boîte de Pétri et le polycarbonate dans des panneaux Axxis (DSM Engineering Products). Comme indiqué dans le tableau 1, PI, PET et PEEK ont des coefficients d'absorption élevés, tandis que les PMMA, PS et PC sont des absorbeurs faibles à température ambiante pour la longueur d'onde laser utilisée. Ceci donne la possibilité d'étudier l'influence de ce paramètre important sur les résultats du microperçage.

\subsection{Vitesse de perçage et profil stationnaire avec l'exemple du PET}

Une expérience typique de microperçage, réalisée après optimisation du faisceau, est illustrée à la figure 4 . Cela demande de nombreuses impulsions. D'après la figure 4A, la vitesse a une valeur initiale (a) qui est caractéristique d'une ablation de surface avec une expansion 3D de la plume (par opposition à une ablation profonde), ensuite la vitesse se réduit à une constante (b) lorsque le trou devient profond, parce que l'absorption de la plume augmente en raison de son expansion 1D et aussi à cause du phénomène de redépôt. Enfin la vitesse tombe à zéro (c) lorsque le profil stationnaire est atteint. D'après le tableau 1 , avec une vitesse de $0,7 \mu \mathrm{m}$.impulsion ${ }^{-1}$ le profil stationnaire est atteint en 7500 impulsions approximativement. Les images intermédiaires B, C, D sont prises respectivement à 3500, 4500, 6000 impulsions. Nous observons l'évolution du front principal d'ablation au fond

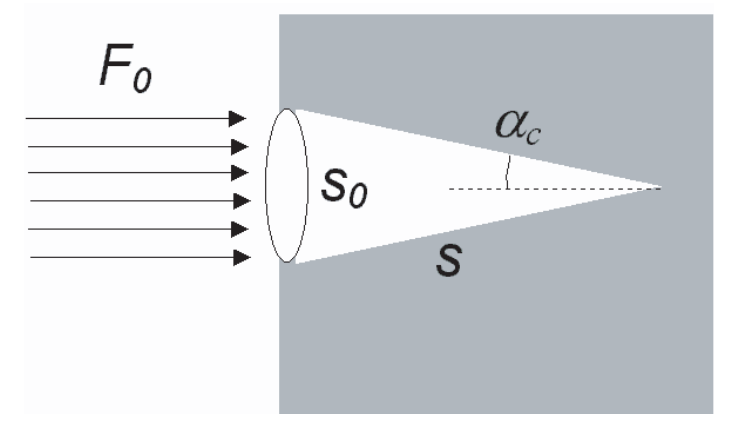

Fig. 5. Modèle simplifié de l'état stationnaire d'un trou conique pour un faisceau idéalement collimaté.

du trou qui est plutôt carré et qui devient plus pointu au fur et à mesure que s'approche la fin du perçage ou l'état stationnaire. Lorsque l'état stationnaire est obtenu, la pointe n'avance plus et les dimensions latérales du trou n'évoluent plus non plus. L'énergie laser est alors piégée à l'intérieur du trou et s'étale sur la surface intérieure du trou de façon à ce que la densité superficielle d'énergie soit inférieure au seuil d'ablation. Ce résultat peut être mieux compris à l'aide du modèle simplifié du trou conique (Fig. 5) (nous verrons ci-dessous que les profils ne sont pas coniques). Un faisceau idéalement et totalement collimaté formerait un cône d'angle $\alpha_{\mathrm{c}}$ et de rapport de forme $1 / \alpha_{\mathrm{c}}$. Le perçage s'arrêterait alors que la fluence sur la paroi tombe à la valeur égale à un certain seuil $F_{\infty}$ de telle manière que :

$$
F_{0} s_{0}=F_{\infty} s=F_{\infty} s_{0} / \alpha_{\mathrm{c}}
$$



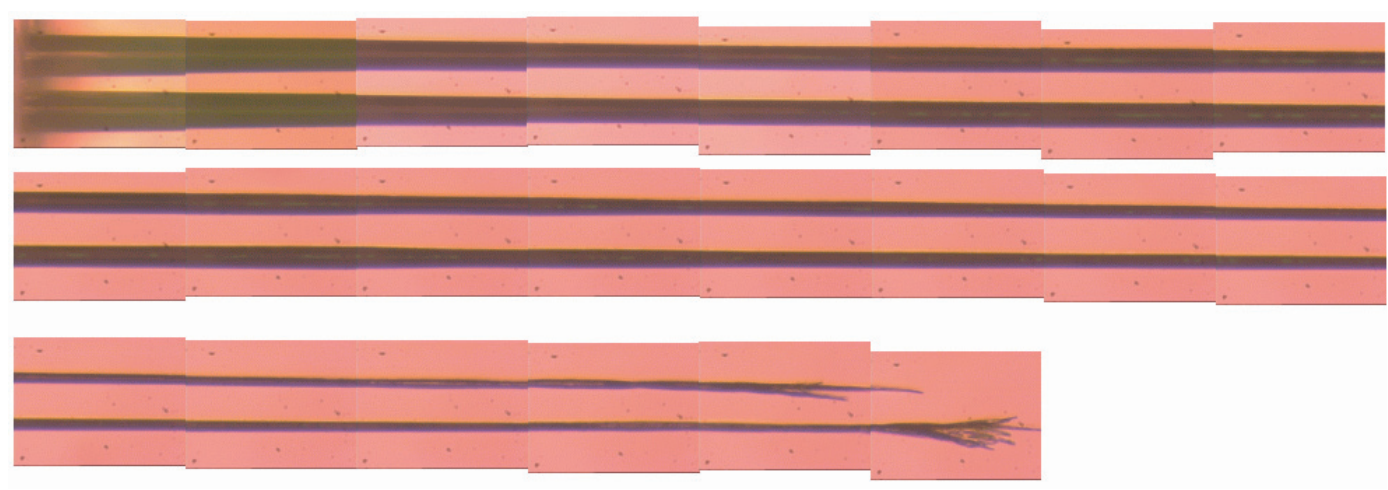

Fig. 6. Paire de trous adjacents dans le polycarbonate, diamètre $25 \mu \mathrm{m}$ et distance de $40 \mu \mathrm{m}$ entre chaque trou, montrant la précision du microperçage laser. Le deuxième perçage n'est pas perturbé par la présence du premier.

À partir de là, le rapport de forme peut être estimé par exemple comme :

$$
R=1 / \alpha=F_{0} / F_{\infty}>F_{0} / F_{\mathrm{t}}
$$

Comme le seuil $F_{\infty}$ auquel le perçage s'arrête est plus bas que le seuil d'ablation habituel $F_{\mathrm{t}}$, avec une fluence typique de $30 \mathrm{~J} . c m^{-2}$ et un seuil d'ablation typique de $30 \mathrm{~mJ} . \mathrm{cm}^{-2}$, un rapport de forme de $10^{3}$ serait prédit. Ceci est comparable à ce que donne l'expérience.

\subsection{Autres polymères}

Le PET sous la forme du Mylar D, présenté ci-dessus donne le meilleur rapport de forme (Tab. 1). Ensuite il y a un groupe avec les PC, PEEK et PI qui présentent un rapport de forme moins élevé $R m \sim 350$ et les PMMA et PS ont des valeurs plus basses. Il est intéressant de comprendre pourquoi lorsque la structure chimique change, les performances de microperçage sont si fortement affectées. Ce point particulier sera discuté ci-dessous lors de la présentation des caractéristiques de perçage spécifiques à chaque polymère.

\subsubsection{Polycarbonate (PC)}

Le polycarbonate donne aussi de très hauts rapports de forme (Tab. 1). La surface après ablation présente une couleur noire caractéristique d'un matériau carboné, déjà observée dans la photoablation de la surface du PC. Comme déjà publié, à un certain point du perçage un phénomène de branchement est observé. Dans la figure 6 il apparaît peu avant la fin du perçage à la pointe finale, lorsque la fluence laser est atténuée à des valeurs au voisinage de la valeur seuil. Le mécanisme de formation des branches [17] est analogue à celui de la formation des cônes, signalés en ablation de surface. Ils se forment parce que la fluence absorbée $F A(i) \cos (i)$ devient égale à $F_{\mathrm{t}}$ ( $i$ est l'angle d'incidence du faisceau). Ce phénomène est spécifique au polycarbonate ainsi qu'à d'autres polymères comme le PI et le PEEK. Ils ont en commun la capacité de former une grande quantité de graphite

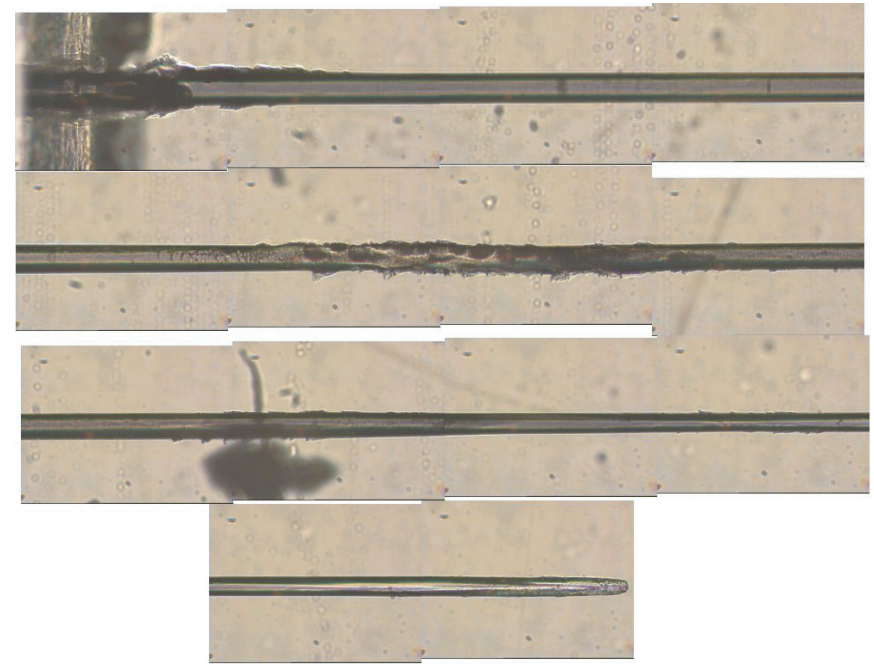

Fig. 7. Trou laser KrF dans le PMMA. Diamètre $60 \mu \mathrm{m}$ et longueur de 6,5 mm $(\mathrm{AR}=100)$. L'entrée est en haut à gauche et la pointe est en bas à droite. L'image est le résultat de l'assemblage d'images partielles.

par ablation, tandis que les autres polymères PET, PS et PMMA beaucoup moins. On pense aussi que les produits d'ablation expulsés sont alors des gaz et des nanoparticules par opposition à des micro- et nano-gouttes dans les autres cas.

\subsubsection{Poly(méthyle métacrylate)}

Un exemple de trou formé par le laser $\mathrm{KrF}$ dans le PMMA est montré à la figure 7 avec un grossissement qui permet de révéler plusieurs caractéristiques intéressantes concernant les mécanismes de perçage. Son rapport de forme est seulement $R=100$ à la figure 7 et pas le rapport de forme maximal du tableau 1 parce que son diamètre ne permet pas d'atteindre la fluence suffisante pour atteindre $R_{\mathrm{m}}$. Un faisceau de plus petit diamètre ( $30-35 \mu \mathrm{m})$ obtenu avec un masque de molybdène de plus petit diamètre $(30 \times 5=150 \mu \mathrm{m})$ fournit le spot le plus énergétique, capable d'atteindre le rapport de 


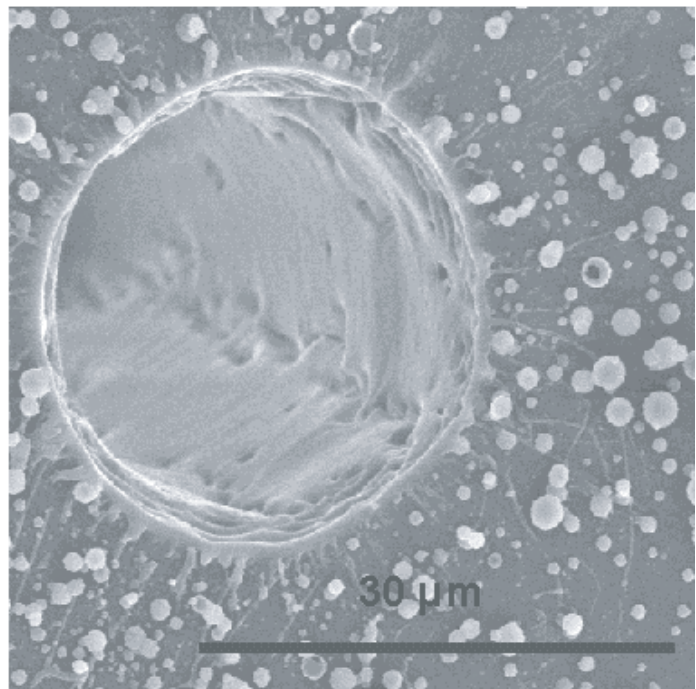

Fig. 8. Image SEM d'un trou dans le PMMA percé au laser KrF. Le diamètre est $30 \mu \mathrm{m}$. On observe de nombreuses gouttelettes redéposées, résultat de l'expulsion de polymère liquide.

forme $R_{\mathrm{m}} \mathrm{du}$ tableau 1 . Une différence principale avec le cas précédent du PC est la forme de la pointe finale, qui est maintenant complètement ronde au lieu d'être ramifiée ou dendritique. Ceci est dû à la présence de PMMA liquide et visqueux pendant l'interaction laser. La tension de surface du liquide produit un ménisque $\mathrm{au}$ fond du trou, qui se re-solidifie entre deux impulsions consécutives. Une telle phase liquide n'est pas produite pendant le perçage des autres polymères PC, PI, PEEK et, comme elle se forme en couche ultrafine [21,22] pendant l'ablation du PET le phénomène semble avoir un effet négligeable sur le microperçage. L'image SEM de la figure 8 montre des gouttelettes de PMMA redéposées autour du trou percé. L'expulsion de liquide peut être un mécanisme important d'enlèvement de matière dans le cas du PMMA. Récemment nous avons aussi démontré les conditions expérimentales dans lesquelles se forment des nanofilaments [23, 24] par expulsion de gouttelettes avec une impulsion laser unique. Dans ce cas la vitesse d'éjection du liquide peut être aussi élevée que $840 \mathrm{~m} . \mathrm{s}^{-1}$ ( Mach 3) d'après notre modèle de la pression de recul, en fonction de la fluence utilisée. Des détails de la surface du trou peuvent être vus dans la figure 7 où, en particulier, quelques micro-cracks sont visibles. Il est difficile d'expliquer leur mécanisme de formation à ce point de l'étude, mais leur présence n'est cependant pas surprenante car le matériau PMMA est exposé à une dose élevée d'UV et les impulsions de $\mathrm{P}$ et $\mathrm{T}$ pendant la formation du trou ont une grande amplitude et imposent de grandes contraintes à la cible. Aussi, à cause de la grande longueur d'absorption $\sim 150 \mu \mathrm{m}$ dans le PMMA (Tab. 1) on imagine que dans le cylindre de la zone affectée autour du trou, les molécules reçoivent une grande quantité d'excitation photonique et thermique. Ce volume a été mesuré par microspectrométrie Raman [25].

\subsection{Recherche du rapport de forme optimal}

Comme il a été indiqué dans le tableau 1, il existe un rapport de forme maximal $R_{\mathrm{m}}$. Il est principalement fonction du type de polymère car le perçage est réalisé avec le même faisceau pour tous les polymères. Mais il est aussi sensible au diamètre du faisceau car la fluence est le paramètre le plus important qui contrôle l'expérience. Nous avons déterminé que le rapport de forme varie en fonction de la fluence comme $: R=R_{\mathrm{m}}\left(1-e^{-F / F_{\mathrm{c}}}\right)$, où $F_{\mathrm{c}}$ est une fluence caractéristique du polymère [13]. Maintenant la fluence au centre du spot est le paramètre le plus important car elle doit être le plus élevée possible afin de percer plus profond comme suggéré par l'équation (3). Elle augmente en diminuant la taille du faisceau, mais à un certain point, la diffraction tend à élargir le faisceau dans un angle solide plus grand. C'est la raison du rapport de forme maximal $R_{\mathrm{m}}$ que nous avons pu trouver dans nos expériences. Il est obtenu avec un diamètre de faisceau de l'ordre de 25-30 $\mu \mathrm{m}$ en fonction du polymère et c'est une limite due aux caractéristiques du faisceau laser.

\section{Modèle}

Le modèle analytique présenté ci-dessous a été décrit en détail dans les publications précédentes [16,17]. Le faisceau laser est approché comme dans la figure 9a par un faisceau collimaté de manière à ce qu'il diverge du point focal $\mathrm{O}$ de la lentille de projection $\mathrm{P}$ et il donne un spot de diamètre $d$ à la surface de l'échantillon S positionné dans le plan image du masque M. Ainsi dans le modèle, $\mathrm{O}$ est une source ponctuelle et virtuelle (Fig. 9b) envoyant ses rayons de lumière sur l'échantillon et dans le trou, dans un cône de demi-angle $\alpha_{0}$. Les paramètres géométriques du laser sont déterminés par le cône de lumière émis par $\mathrm{O}$ dans la direction du trou et défini par la distance $z_{0}$, l'angle du cône $\alpha_{0}$ (divergence, ouverture numérique du faisceau) et le profil de fluence (chapeau carré, gaussien...) qui est choisi comme une fonction de l'inclinaison $\alpha$ du rayon particulier (Fig. 9b). Nous montrons par le présent modèle que la géométrie du trou est prédéterminée lorsque ces paramètres sont fixés. Le modèle a été validé en ajustant la prédiction théorique $l(F)$ à la longueur expérimentale des trous. Pour des raisons liées au diamètre du trou dépendant de la dose, au profil stationnaire, le rapport de forme $R$ n'est pas utilisé pour cet ajustement.

\subsection{Condition du profil stationnaire}

À la fin du perçage le profil du trou est stationnaire et ainsi ne change plus notablement car l'ablation ne se produit plus. À ce point cela veut dire que la fluence laser absorbée à la surface du trou $F_{\text {a }}$ est devenue plus petite que la valeur seuil que nous avons appelée $F_{\infty}$ et constante 

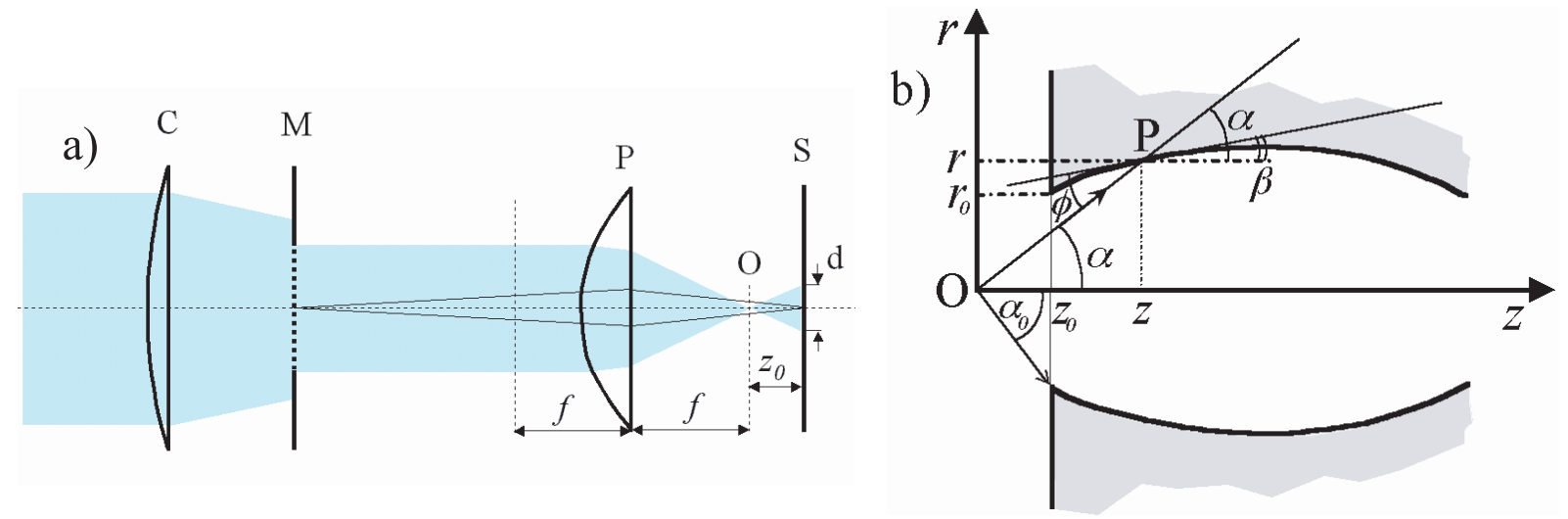

Fig. 9. a) Schéma du banc optique utilisé dans la modélisation du microperçage par laser. b) Modèle de source ponctuelle virtuelle avec propagation des rayons à l'intérieur du trou en perçage. O est une source ponctuelle et virtuelle à la distance $z_{0}$ de l'échantillon et émettant des rayons dans un cône d'angle $\alpha_{0}$ vers l'intérieur du trou avec une intensité variant selon l'inclinaison $\alpha$ (profil laser). Le diamètre du trou est très exagéré dans le but d'une meilleure visualisation.

sur toute la surface. Au point $\mathrm{P}$ de la figure $9 \mathrm{~b}$ nous avons la condition :

$$
F_{\mathrm{a}}(r, z)=F_{\infty}
$$

C'est l'équation qui caractérise le profil stationnaire. $F_{\infty}$ est le seuil de fluence à laquelle l'ablation stoppe. Il dépend principalement du polymère et de sa modification introduite par la dose laser délivrée pendant le processus de perçage. Nous verrons qu'il est fondamentalement différent du seuil d'ablation bien connu. C'est simplement le seuil en dessous duquel l'arrachement de la matière ne peut plus se produire.

\subsection{Profil du trou au stade stationnaire}

La fluence absorbée au point $\mathrm{P}$ de coordonnées $(r, z)$ du profil (Fig. 9b) est donnée par :

$$
F_{\mathrm{a}}(r, z)=A_{\mathrm{eff}}(\phi(r, z)) \times F(r, z) \sin \phi(r, z)
$$

où $F(r, z)$ est la fluence incidente au point $\mathrm{P}$ et $\Phi(r, z)$ est l'angle d'incidence pour le rayon OP. $A_{\text {eff }}(\phi(r, z))$ est le coefficient d'absorption effectif local pour la radiation laser au point P. $F(r, z)$ est donnée par la distribution angulaire $F(\alpha)$ à $z=z_{0}$

$$
F(r, z)=F(\alpha) z_{0}^{2} / z^{2}
$$

dans laquelle le facteur $z_{0}^{2} / z^{2}$ décrit l'atténuation de la fluence avec la distance $z$. Les deux raisons principales de l'arrêt de l'ablation au profil stationnaire sont : l'atténuation grandissante avec la distance et l'inclinaison croissante de la paroi absorbante au fur et à mesure de la progression du perçage. Dans l'équation (5) $\Phi(r, z)$ peut s'exprimer en fonction de l'inclinaison des rayons $\alpha$ (Fig. 9b) :

$$
\phi(r, z)=\alpha(r, z)-\beta(r, z)
$$

où $\alpha=\operatorname{arctg}(r / z) \cong r / z$ comme $r$ est petit et :

$$
\beta=\operatorname{arctg}(\mathrm{d} r / \mathrm{d} z) \cong \mathrm{d} r / \mathrm{d} z=\mathrm{d}(\alpha z) / \mathrm{d} z=\alpha+z(\mathrm{~d} \alpha / \mathrm{d} z)
$$

est l'inclinaison locale de la paroi au point P. En substituant (8) dans (7) il vient:

$$
\sin \phi(r, z) \cong \phi(r, z) \cong-z(\mathrm{~d} \alpha / \mathrm{d} z) .
$$

En combinant (5), (6) et (9) l'équation différentielle déterminant le profil est obtenue :

$$
z \frac{\mathrm{d} z}{\mathrm{~d} \alpha}=-A_{\mathrm{eff}}(\alpha) \frac{z_{0}^{2}}{F_{\infty}} F(\alpha)
$$

Maintenant dans ce qui suit $A_{\text {eff }}$, le coefficient effectif d'absorption, aura la valeur 1 [17] sur la base des arguments suivants. La radiation laser déjà entrée dans le trou a très peu de chance de ressortir vers l'extérieur et donc un mécanisme de diffusion multiple se produit à l'intérieur du trou ce qui fait que l'absorption de l'énergie est totale. Ainsi en chaque point les rayons incidents sont finalement absorbés quelle que soit leur origine puisque la diffusion multiple domine la propagation du faisceau à l'intérieur du trou. De plus les tentatives d'ajustement du modèle aux points expérimentaux, en prenant toute autre valeur que $A_{\text {eff }}=1$, ne réussissent pas à fournir la bonne prévision des résultats expérimentaux. Avec cette valeur 1, l'équation (10) du modèle de réflexion multiple peut être résolue sous forme paramétrique $r(\alpha)$, $z(\alpha)$ avec $\alpha$, l'inclinaison des rayons, comme paramètre. L'équation du profil du trou au régime stationnaire final est alors donnée par :

$$
r(\alpha)=\alpha z(\alpha), \quad z(\alpha)=z_{0}\left[1+\frac{2}{F_{\infty}} \int_{\alpha}^{\alpha \infty} F\left(\alpha^{\prime}\right) \mathrm{d} \alpha^{\prime}\right]^{1 / 2}
$$

où $\alpha_{\infty}$ est l'inclinaison limite au-dessus de laquelle l'intégration est négligeable. La longueur du trou (ou sa profondeur) est déduite de (11) pour la valeur du paramètre $\alpha=0$ :

$$
\begin{aligned}
l(F) & =z(\alpha=0)-z_{0} \\
& =z_{0}\left[\left[1+\frac{2}{F_{\infty}} \int_{0}^{\alpha_{\infty}} F\left(\alpha^{\prime}\right) \mathrm{d} \alpha^{\prime}\right]^{1 / 2}-1\right]
\end{aligned}
$$




\subsection{Ajustement des profondeurs expérimentales avec le profil « chapeau carré »}

Comme expliqué plus haut le rapport de forme du trou n'a pas été choisi comme grandeur expérimentale pour l'ajustement, car il sature à forte fluence à cause de sa légère augmentation avec la fluence en régime stationnaire, ce qui ne peut pas être prédit simplement par ce modèle. Si un diamètre constant avec la fluence est choisi pour calculer le rapport de forme, alors, comme la longueur (12) augmente avec la fluence, le rapport de forme obtenu ne reproduit pas la saturation observée dans l'expérience. Au contraire, nous avons trouvé que la longueur du trou augmente bien avec la fluence comme prédit par l'équation (12) et montré à la figure 10. Les résultats expérimentaux ont été ajustés avec succès à l'aide du modèle (12) pour les six polymères de ce travail. Le profil « chapeau carré » est défini par :

$$
F(\alpha)=\text { const. }=F
$$

pour $0<\alpha<\alpha_{0}$ (voir Fig. 9b) et la longueur du trou en fonction de la fluence devient :

$$
l(F)=z_{0}\left[\left[1+2\left(\frac{F}{F_{\infty}}\right)\left(\frac{r_{0}}{z_{0}}\right)\right]^{1 / 2}-1\right]
$$

où apparaissent les rapports $r_{0} / z_{0}=\alpha_{0}$ déjà mentionné ci-dessus et qui représente l'ouverture numérique du faisceau laser, et $F / F_{\infty}$ un rapport qui peut être appelé la surfluence, très important car il doit être aussi large que possible pour fournir un perçage profond. La longueur expérimentale du trou en fonction de la fluence est reproduite avec succès par l'équation (14) avec $F_{\infty}$ comme seul paramètre d'ajustement pour chaque polymère du tableau 2. Dans l'équation (14) on voit que la surfluence $F / F_{\infty}$, nombre sans dimension, joue un rôle particulier car seules les valeurs élevées donnent des bonnes longueurs de perçage, qui assurément conduisent aux rapports de forme élevés obtenus dans ce travail (e.g. pour le PET $F / F_{\infty}=3 \times 10^{4}$ avec $F_{\infty}=1 \mathrm{~mJ} . \mathrm{cm}^{-2}$ et une fluence laser de $F=30{\mathrm{~J} . \mathrm{cm}^{-2}}^{-2}$. Si le profil du faisceau est pris gaussien [17]

$$
F(\alpha)=F \exp \left(-2 \alpha^{2} / w_{\alpha}^{2}\right)
$$

la longueur du trou en fonction de la fluence est seulement légèrement modifiée :

$$
l(F)=z_{0}\left[\left[1+2\left(\frac{F}{F_{\infty}}\right)\left(\frac{\sqrt{\pi} w_{\alpha}}{\sqrt{2}}\right)\right]^{1 / 2}-1\right]
$$

$w_{\alpha}$ est le paramètre de la gaussienne.

\subsection{Profil du trou au stade stationnaire}

À l'aide de l'équation (11), le profil calculé du trou peut être tracé comme dans la figure 11, pour un faisceau de profil « chapeau carré » avec différentes valeurs

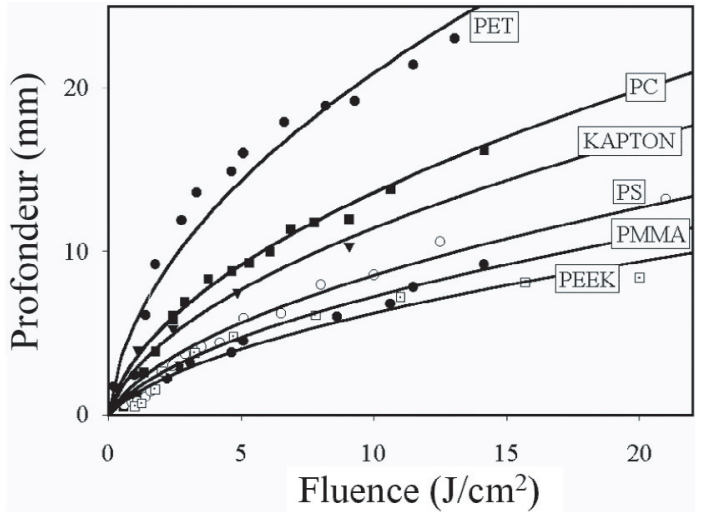

Fig. 10. Longueur du trou au profil stationnaire en fonction de la fluence. Les points sont les mesures expérimentales et les lignes continues sont obtenues avec l'équation (14) du modèle, en ajustant $F_{\infty}$.

Tableau 2. Seuils caractéristiques $F_{\infty}$ de fin d'ablation et fin de perçage fournis par le modèle de l'équation (14).

\begin{tabular}{cc}
\hline Matériau & $F_{\infty}\left(\mathrm{mJ} . \mathrm{cm}^{-2}\right)$ \\
\hline PET & 1 \\
PC & 2,2 \\
PEEK & 8,5 \\
PI & 3 \\
PS & 5 \\
PMMA & 6,6 \\
\hline
\end{tabular}

de la fluence. Si la fluence est exprimée en unité de $F_{\text {par }}=F_{\infty} z_{0} / r_{0}$, qui est une valeur particulière pour laquelle à l'entrée du trou $r=r_{0}$ les parois latérales sont parallèles (Fig. 11 courbe (2)). Les profils calculés dans la figure 11 peuvent être aussi bien convergents, surtout au fond, que divergents, surtout à l'entrée, suivant la valeur de $F$ (profils 3-5 par exemple). Dans ce modèle de réflexion multiple tous les rayons émis par la source ponctuelle virtuelle et se propageant sur les parois latérales, sont totalement absorbés $\left(A_{\mathrm{eff}}=1\right)$. En conséquence pour la transmission, la seule contribution est donnée par les rayons qui ne sont pas bloqués par les parois à la distance considérée. Pour un profil « chapeau carré» l'énergie transmise $E(z)$ est simplement directement proportionnelle à l'angle solide correspondant à la sortie $z$ vu par la source $\mathrm{O}$ et la transmission suivante est obtenue :

$$
T(z)=\frac{E(z)}{E\left(z_{0}\right)}=\frac{\pi r(z)^{2} z_{0}^{2}}{\pi r_{0}^{2} z^{2}}
$$

D'après la figure 12 on constate que la transmission calculée est très proche d'une fonction linéaire de la profondeur, ce qui reproduit très bien les mesures expérimentales [17].

\section{Mécanismes et perspectives}

Le présent modèle est une bonne base théorique pour expliquer les caractéristiques du perçage observées 


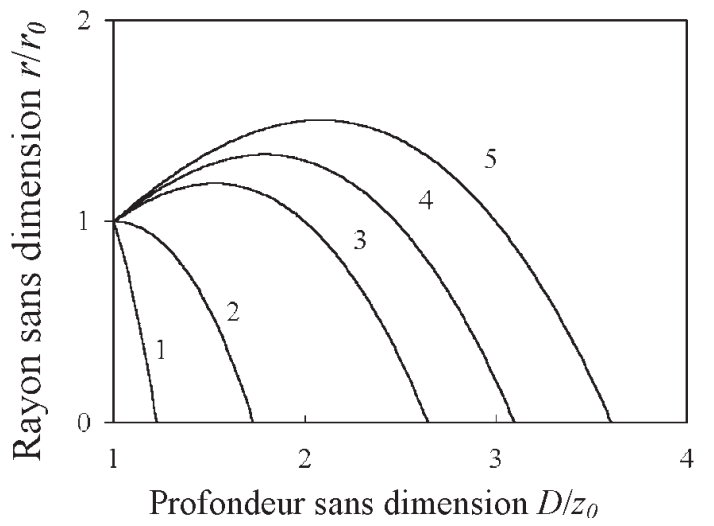

Fig. 11. Profils stationnaires calculés pour un certain nombre de fluences laser, $D=z-z_{0} . F / F_{\text {par }}=:$ (1) 0,25 ; (2) 1 ; (3) 3 ; (4) 4,3 ; (5) $6 . F_{\text {par }}=F_{\infty} z_{0} / r_{0}$ est une fluence particulière qui donne des parois parallèles à l'entrée du trou.

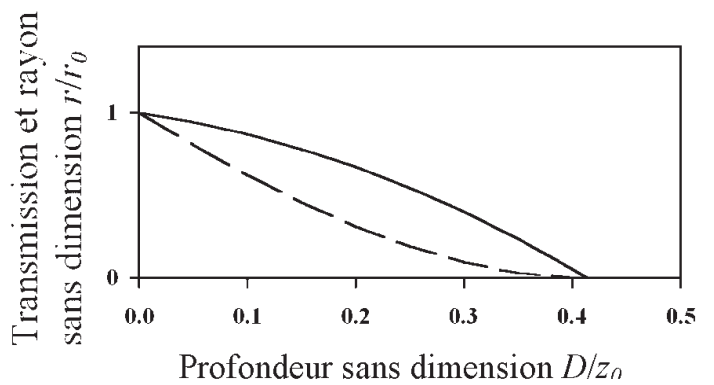

Fig. 12. Transmission (pointillé) en fonction de la profondeur sans dimension, pour un profil carré $F=3 F_{\text {par }}$.

expérimentalement. En particulier, il prédit les grandeurs géométriques du trou percé par laser lorsqu'il a atteint son profil stationnaire final. Il apporte une réponse raisonnable à la question qui a été le point de départ de ce travail, c'est-à-dire jusqu'à quelle profondeur un laser peut-il percer? Il définit une surface contour $r(\alpha), z(\alpha)$ où $F_{\mathrm{a}}(\alpha)=F_{\infty}$ et à l'intérieur de laquelle le polymère est enlevé par ablation (volume ablatif). Il est important de souligner qu'il est indépendant du caractère pulsé de la radiation laser. Il est donc applicable aussi bien au perçage par laser continu à condition que le mécanisme d'enlèvement de matière implique seulement une transition solide-vers-gaz dans le volume absorbant, lorsque la fluence est plus grande que le minimum nécessaire pour l'arrachement de matière $F_{\infty}$. Par contre il n'est pas conçu pour expliquer comment le perçage se réalise dans ses étapes intermédiaires. Dans le présent perçage par laser pulsé, le processus est répétitif avec dans chaque période la séquence d'évènements suivante : absorption, excitation, ablation et refroidissement. À la fréquence de notre laser $(200 \mathrm{~Hz})$ on considère que le refroidissement est complet entre deux impulsions. Cela veut dire que la température retourne à l'ambiante car la chaleur diffuse suffisamment rapidement (le temps de refroidissement de la surface est de l'ordre de 1-10 ms) autour du trou dans un volume strictement limité à celui que peut atteindre le contenu énergétique d'une seule impulsion, c'est-à-dire seulement $\sim 1-10^{2} \mu \mathrm{m}$ dans la plupart des cas. L'étape d'absorption se produit en général sur une surface refroidie pour chaque nouvelle impulsion et à cause de l'affaiblissement de l'énergie transmise lorsque la profondeur augmente, la fin du perçage se produit lorsque pour chaque point de la surface $F_{a}(r, z)=F_{\infty}$. Après quelques instants de perçage, lorsque l'ablation dans le matériau devient profonde, le redépôt de matière ablatée entre en jeu plus ou moins en fonction de la nature du polymère et des mécanismes. Par exemple le PMMA s'ablate typiquement par un mécanisme thermique avec la formation d'une grande quantité de liquide, qui est expulsé sous la forme de gouttelettes ou redéposé sur les parois. Le processus de redépôt suivi de la réablation est une des deux raisons principales qui réduisent la vitesse (Fig. 4A). L'autre raison est l'écrantage de la plume qui est augmenté à cause de l'expansion 1D de l'ablation profonde à la place de la $3 \mathrm{D}$ de l'ablation de surface. De plus à ce niveau de fluence, cette plume en expansion et absorbant fortement, probablement dans un état de plasma transitoire, joue aussi le rôle de stockage de l'énergie incidente et peut la redonner au matériau pendant l'expansion. Après chaque impulsion le transfert d'énergie par le plasma vers la surface peut avoir un effet d'ablation ou de décapage pour les produits redéposés. Il est aussi probable que si un film liquide est formé sur les parois intérieures pendant le perçage, il est accéléré vers la sortie par la quantité de mouvement transférée par la plume en mouvement vers l'extérieur. Ceci peut aussi contribuer à accroître le diamètre du trou. La nature de l'interaction laser/matière est principalement laser-surface mais peut être aussi laser-plasma et plasma-surface avec, pour le dernier, une délocalisation sur la surface du trou toute entière. Jusqu'ici les connaissances sur les mécanismes intimes de transport vers l'extérieur lorsque l'ablation est profonde, sont insuffisantes et incomplètes.

Un bon contrôle du faisceau laser conduisant au perçage profond est aussi souhaitable pour de nombreuses approches d'usinage laser. Un dispositif expérimental analogue au nôtre peut être utilisé pour une découpe précise et compliquée sur une grande profondeur à l'aide d'un déplacement relatif programmé de la cible par rapport au faisceau : voir l'exemple figure 13 dans la référence [13], et le perçage hélicoïdal dans la référence [5]. Le perçage massif de matrice de trous est aussi nécessaire pour certaines applications. Des lasers à haute fréquence [26] (jusqu'à $5-50 \mathrm{kHz}, 10 \mathrm{ps}-30 \mathrm{~ns}, I=10-20 \mathrm{MW}$ par exemple) deviennent disponibles et ont un grand intérêt si on recherche une grande vitesse d'usinage. Les courtes longueurs d'onde du laser à excimère (193 nm et $248 \mathrm{~nm}$ ) sont préférables dans la plupart des cas, mais les harmoniques $4 \omega$ et $3 \omega$ du laser Nd/YAG sont des sources intéressantes avec les fréquences les plus élevées. De tels lasers à fréquence élevée peuvent en principe offrir des vitesses d'usinage élevées. Néanmoins de nouveaux problèmes d'accroissement de température peuvent apparaître à fort taux de répétition [26].

D'autres matériaux comme les métaux, céramiques et verres peuvent être aussi traités de manière similaire à 
l'aide du faisceau du laser $\mathrm{KrF}$ [25]. Cependant à cause des seuils d'ablation plus élevés $\left(F_{\mathrm{t}}\right.$ et $\left.F_{\infty}\right)$ des rapports de forme beaucoup plus modestes sont obtenus : 10-50 suivant le matériau. De plus l'intérêt d'utiliser un laser ultraviolet est moins évident dans ce cas. D'autres sources de longueur d'onde visible sont utilisables pour ces matériaux, en particulier le laser à vapeur de cuivre [27]. Aussi des travaux intéressants sont réalisés avec des lasers femtoseconde car des ablations sans liquide ont été rapportées dans les métaux [28,29], silice [30], le quartz [31], et les polymères [32]. Le microperçage des polymères par laser femtoseconde ultraviolet est encore d'un intérêt fondamental et reste une perspective d'avenir.

\section{Conclusions}

Nous avons présenté des travaux expérimentaux et théoriques récents sur le microperçage à haut rapport de forme dans les polymères avec un faisceau laser $\mathrm{KrF}$. Un bon faisceau est obtenu en condensant le laser sur un masque circulaire de molybdène qui est imagé avec une lentille de projection de courte focale sur la surface de la cible. Le positionnement de la surface à micropercer, précis à $\pm 50 \mu \mathrm{m}$, est obtenue en superposant au masque, une grille fine de nickel et en recherchant son image par des ablations successives à différentes positions (tous les $100 \mu \mathrm{m})$. Le meilleur rapport de forme $\sim 600$ est obtenu dans le PET pour un trou de $25-30 \mu \mathrm{m}$ de diamètre et de $18 \mathrm{~mm}$ de longueur. Six polymères différents ont été étudiés. La dépendance de la longueur du trou en fonction de la fluence est prédite avec succès par notre nouveau modèle, qui fournit aussi un nouveau seuil $F_{\infty}$, l'énergie minimale nécessaire pour l'arrachement de matière après irradiation avec un grand nombre d'impulsions. Un paramètre important pour le perçage profond est la surfluence, rapport $F_{0} / F_{\infty}$ qui détermine la profondeur à laquelle le profil de perçage devient stationnaire. Ces résultats trouveront des applications nombreuses dans des recherches futures.

Remerciements. La recherche est financée en partie par la Région Aquitaine et les Projets FEDER. Les auteurs remercient le CNRS (Centre National de Recherche Scientifique) pour un contrat de recherche pour VNT, qui est détaché de l'Institut de Physique Générale de Moscou.

\section{Références}

[1] B. Braren, R. Srinivasan, Optical and photochemical factors which influence etching of polymers by ablative photodecomposition, J. Vac. Sci. B 3 (1985) 913-917

[2] R.W. Olson, W.C. Swope, Laser drilling with focused Gaussian beams, J. Appl. Phys. 72 (1992) 3686-3696

[3] M. Olfert, W.W. Duley, Holographic interferometry of isotherms during laser drilling of fused quartz, J. Phys. D: Appl. Phys. 29 (1996) 1140-1145

[4] J.A. Clarke, T.S. Givens, J.T. Williamson, Laser drilling of diesel fuel injector nozzles, Lambda Highlights 62 (2003) 4-5
[5] F. Dausinger, H. Hügel, V. Konov, Micro-machining with ultrashort laser pulses : from basic understanding to technical applications, Proc. SPIE 5147 (2003) 106

[6] C. Körner, R. Mayerhofer, M. Hartmann, H.W. Bergmann, Physical and material aspects in using visible laser pulses of nanosecond duration for ablation, Appl. Phys. A 63 (1996) 123

[7] C. Paterson, A.S. Holmes, R.W. Smith, Excimer laser ablation of microstructures : A numerical model, J. Appl. Phys. 86 (1999) 6538

[8] A. Luft, U. Franz, A. Emsermann, J. Kaspar, A study of thermal and mechanical effects on materials induced by pulsed laser drilling, Appl. Phys. A 63 (1996) 93

[9] Y. Chen, H.Y. Zheng, K.S. Wong, S.C. Tam, Excimer laser drilling of polymers, Proc. SPIE 3184 (1997) 202

[10] N. Rykalin, A. Uglov, A. Kokora, Laser Machining and Welding (MIR, Moscou 1978)

[11] C. Lehane, H.S. Kwok, Enhanced drilling using a dualpulse Nd :YAG laser, Appl. Phys. A 73 (2001) 45

[12] T. Otani, L. Herbst, M. Heglin, S.V. Govorkov, A.O. Wiessner, Microdrilling and micromachining with diodepumped solid-state lasers, Appl. Phys. A 79 (2004) 1335

[13] S. Lazare, J. Lopez, F. Weisbuch, High-aspect-ratio microdrilling in polymeric materials with intense $\mathrm{KrF}$ laser radiation, Appl. Phys. A 69 (1999) S1

[14] J. Lopez, S. Lazare, Microperçage des polymères par laser à excimère, J. Phys. IV France 9 (1999) 153

[15] J. Lopez, S. Lazare, F. Weisbuch, High aspect ratio microdrilling in polymeric materials with intense $\mathrm{KrF}$ laser radiation, Proc. SPIE 3822 (1999) 77

[16] V.N. Tokarev, J. Lopez, S. Lazare, Modelling high aspect ratio microdrilling of polymers with UV laser ablation, Appl. Surf. Sci. 168 (2000) 75

[17] V.N. Tokarev, J. Lopez, S. Lazare, F. Weisbuch, High aspect ratio microdrilling of polymers with UV laser ablation: experiment with analytical model, Appl. Phys. A 76 (2003) 385

[18] A.E. Siegman, Lasers, 6th ed., University Science Book, 1986

[19] S. Lazare, V. Tokarev, Recent experimental and theoretical advances in microdrilling of polymers with ultraviolet laser beams, conférence invitée présentée au "Fifth International Symposium on Laser Precision Microfabrication, LPM04" (Nara, Japon, mai 2004), et publié en anglais dans I. Miyamoto, H. Helvajian, K. Itoh, K.F. Kobayashi, A. Ostendorf, K. Sugioka, (ed.), Proceedings of SPIE 5662 (2004) 221-231

[20] F. Weisbuch, S. Lazare, F.N. Goodall, D. Débarre, Submicron resolution ablation with a KrF excimer laser beam patterned with a projection lens, Appl. Phys. A 69 (1999) 413

[21] S. Lazare, P. Benet, Surface amorphization of Mylar films with the excimer laser radiation above and below ablation threshold : ellipsometric measurements, J. Appl. Phys. 74 (1993) 4953

[22] F. Weisbuch, V.N. Tokarev, S. Lazare, D. Débarre, Ablation with a single micropatterned $\mathrm{KrF}$ laser pulse : quantitative evidence of transient liquid microflow driven by the plume pressure gradient at the surface of polyesters, Appl. Phys. A 76 (2003) 613

[23] F. Weisbuch, V.N. Tokarev, S. Lazare, C. Belin, J.L. Bruneel, Millimeter long nanofibers of PMMA spun at super high speed by ablation with one pulse of the $\mathrm{KrF}$ excimer laser, Appl. Phys. A 75 (2002) 677 
[24] F. Weisbuch, V.N. Tokarev, S. Lazare, C. Belin, J.L. Bruneel, Millimeter long PMMA nanofibers - A new form of material removal in laser ablation, Thin Solid Films 453-454 (2004) 394

[25] J. Lopez, Ablation profonde et microperçage de matériaux polymères par laser à excimères, Thèse de doctorat de l'Université de Bordeaux 1, 1997

[26] E.G. Gamaly, A.V. Rode, B. Luther-Davis, Ultrafast ablation with high-pulse-rate lasers. Part I : Theoretical considerations, J. Appl. Phys. 85 (1999) 4213

[27] M.R.H. Knowles, Micro-ablation with high power pulsed copper vapor laser, Opt. Expr. 7 (2000) 50

[28] G. Kamlage, T. Bauer, A. Ostendorf, B.N. Chichkov, Deep drilling of metals by femtosecond laser pulses, Appl. Phys. A 77 (2003) 307
[29] S. Juodkazis, H. Okuno, N. Kujime, S. Matsuo, H. Misawa, Hole drilling in stainless steel and silicon by femtosecond pulses at low pressure, Appl. Phys. A 79 (2004) 1555

[30] L. Shah, J. Tawney, M. Richardson, K. Richardson, Femtosecond laser deep hole drilling of silicate glasses in air, Appl. Surf. Sci. 183 (2001) 151

[31] H. Varel, D. Ashkenasi, A. Rosenfeld, M. Wähmer, E.E.B. Campbell, Micromachining of quartz with ultrashort laser pulses, Appl. Phys. A 65 (1997) 367

[32] Y. Zhang, R.M. Lowe, E. Harvey, P. Hannaford, A. Endo, High aspect ratio micromachining of polymers with ultrafast laser, Appl. Surf. Sci. 186 (2002) 345 